\title{
Vitamin C und Krebs - hat Pauling doch Recht?
}

Wenn "Spiegel online" oder »BBC News" und viele andere populäre Magazine sich der Ergebnisse einer wissenschaftlichen Studie (1) auf breiter Front annehmen, die Fachwelt die Ergebnisse aber weitgehend totschweigt, dann kann man davon ausgehen, dass diese Resultate

Ascorbinsäure kann Krebszellen selektiv zerstören irgendwie nicht ins Konzept passen.

Die Reaktionen der etablierten "Scientific Community" sehen dann meist so aus:

1. Das Studiendesign war für die Fragestellung nicht geeignet.

2. Das Datenmanagement und die verwendeten statistischen Verfahren waren verfehlt.

3. Die Interpretation der Ergebnisse durch die Autoren ist überzogen.

4. Die Institution, aus der die Studie kommt, arbeitet nicht professionell.

5. Das Journal, in dem die Studie veröffentlich wurde, validiert nicht ausreichend.

6. Es handelt sich um eine exotische Studie, außerhalb des anerkannten Mainstreams.

7. etc. etc.

Zusammengefasst: Die Evidenzstufe ist gering, die Studie verschwindet im üblichen wissenschaftlichen Informations-Chaos und bleibt ohne nachhaltige Wirkung.

"Bedauerlicherweise" kann das wissenschaftliche Establishment nun für die genannte Studie der Arbeitsgruppe von Mark Levine keines der oben erwähnten "Killer-Argumente" anführen. Levine ist als Wissenschaftler absolut anerkannt, die National Institutes of Health in Bethesda genießen größtes Ansehen, die Proceedings der National Academy of Sciences gehören zu den besten wissenschaftlichen Journalen, das Studiendesign kann kaum kritisiert werden, das Datenmanagement ist o.k. und die Ergebnisse werden nicht überinterpretiert.
Damit muss nun ganz offenbar die Tatsache akzeptiert werden, dass Ascorbinsäure in millimolaren Konzentrationen verschiedene Krebszellen selektiv zerstören kann. Wichtig ist dabei die Selektivität, d.h. unter eben diesen Bedingungen millimolarer Konzentrationen an Ascorbinsäure werden normale Zellen nicht zerstört.

Dass diese Studie exotischen Charakter hat und allein steht, kann ebenfalls nicht behauptet werden, da ein möglicher Wirkungsmechanismus der Ascorbinsäure auf Krebszellen bereits 2003 von der Arbeitsgruppe von Peter Ratcliffe in Oxford in Cancer Research publiziert wurde (2). Er konnte am Beispiel von Prostatakarzinomzellen zeigen, dass die vom Transkriptionsfaktor HIF (Hypoxie-Induzierbarer Faktor) abhängigen Reaktionen, die das Tumorwachstum steigern, die Angiogenese begünstigen, den glykolytischen Stoffwechsel verstärken etc., durch Ascorbinsäure gehemmt werden, indem Ascorbinsäure durch Hydroxylierung von HIF dessen Abbau begünstigt.

Die Geschichte von Vitamin C und Krebs ist also ganz im Sinne von Pauling keineswegs zu Ende sondern erlebt eher eine Renaissance.

1 Q. Chen, M.G. Espey, M.C. Krishna, J.B. Mitchell, C.P. Corpe, G.R. Buettner, E. Shacter, M. Levine: Pharmacological ascorbic acid concentrations selectively kill cancer cells. PNAS 2005; 102: 13604-13609.

2 H.J. Knowles, R.R. Raval, A.L. Harris, P.J. Ratcliffe: Effect of ascorbate on the activity of hypoxia-inducible factor in cancer cells. Cancer Research 2003; 63: 1764-1768.

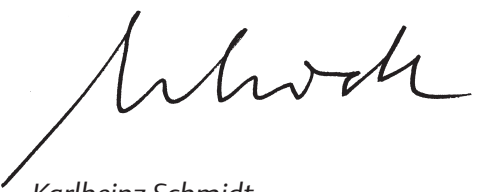

Karlheinz Schmidt 